\title{
Unreduced gametes and neopolyploids in natural populations of Achillea borealis (Asteraceae)
}

\author{
J Ramsey \\ Department of Biology, University of Washington, Seattle, WA, USA
}

\begin{abstract}
Polyploidy is a major mechanism of speciation and adaptation, yet little is known about the origins of polyploids in natural species. I investigated gametic nonreduction and neopolyploid formation in natural tetraploid populations of Achillea borealis (Asteraceae), an autopolyploid complex consisting of tetraploid and hexaploid cytotypes. Cytological analyses of tetraploid populations revealed the occurrence of reduced $(n=2 x)$ as well as unreduced 'big' $(2 n=4 x)$ and 'jumbo' $(4 n=8 x)$ pollen grains, which were clearly distinguished by size. Production of unreduced pollen was monitored in two tetraploid populations in 1997 and 1998. Mean population-level frequencies of unreduced pollen ranged from 0.030 to $0.538 \%$, with as few as one-third and as many as one-half of sampled plants producing unreduced grains. Eight individuals were found to produce $>1 \%$
\end{abstract}

unreduced pollen, with highest observed frequencies of 7.0, 13.2 and 15.8\%. Experimental crosses using high unreduced pollen producers as male parents generated viable seeds. However, the frequency of neohexaploids in the progeny of experimental crosses $(0.388 \%)$ was similar to that observed in progeny of randomly selected, openpollinated control parents $(0.465 \%)$. These results suggest that unreduced eggs are the most likely source of new polyploids. In spite of the inefficiency of unreduced pollen in unilateral sexual polyploidization, the overall rate of neohexaploid formation (one in 233) was several orders of magnitude greater than estimates of genic mutation rates.

Heredity (2007) 98, 143-150. doi:10.1038/sj.hdy.6800912; published online 8 November 2006

Keywords: chromosome evolution; genome duplication; mutation; polyploidy; sexual polyploidization; unreduced gametes

\section{Introduction}

Polyploidy, the possession of three or more chromosome complements, is common in plants. It is estimated that between one-half and two-thirds of flowering plants are polyploid (Grant, 1981; Masterson, 1994). Diversification within ancient polyploid lineages has contributed to the prominence of polyploidy, but ploidy differences distinguish many related congeners and even populations within species (Lewis, 1980). Moreover, formation of polyploids is ongoing in many taxa (Ramsey and Schemske, 1998), and phylogenetic studies indicate that polyploid species often have multiple historical origins (Soltis and Soltis, 1999). Polyploids exhibit distinctive morphological, physiological and life-history characteristics that may allow plants to colonize new habitats or expand their geographic range (Müntzing, 1936; Clausen et al., 1945; Levin, 1983; Otto and Whitton, 2000; Ramsey and Schemske, 2002). Because cytotypes are reproductively isolated by strong postzygotic barriers, polyploidy is also regarded as a major mechanism of speciation in flowering plants (Coyne and Orr, 2004).

In spite of its importance, the factors contributing to polyploid evolution are poorly understood. Characterization of polyploid formation and establishment will require

Correspondence: Professor J Ramsey, Department of Biology, University of Rochester, 213 Hutchison Hall, River Campus, Rochester, NY 14627-0211, USA.

E-mail: justin_ramsey@mac.com

Received 9 February 2006; revised 30 August 2006; accepted 12 September 2006; published online 8 November 2006 detailed cytological investigations of polyploid origins coupled with ecological studies of neopolyploids and established cytotypes in natural plant populations (Thompson and Lumaret, 1992). Studies of agricultural systems point to recurrent polyploid formation in plant populations via gametic nonreduction (Harlan and deWet, 1975; Bretagnolle and Thompson, 1995; Ramsey and Schemske, 1998). However, the occurrence of unreduced gametes and neopolyploids has rarely been documented in nature and there are few published estimates of rates of neopolyploid formation in natural systems.

Here, I combine microscopy techniques with flow cytometry to investigate polyploid origins in Achillea borealis (Asteraceae), a taxonomic species consisting of tetraploid and hexaploid populations. Specifically, I examine the cytological character of unreduced pollen and the frequency of unreduced pollen production in two natural tetraploid populations. The efficiency of unreduced pollen in unilateral sexual polyploidization is tested by comparing frequencies of neohexaploids in experimental crosses involving tetraploid unreduced pollen producers vs 'background' frequencies in randomly selected, open-pollinated maternal sibships. Finally, an overall rate of autohexaploid formation is estimated.

\section{Methods}

Study system

Achillea (Anthemidae-Asteraceae) is a large genus of perennial herbs (Clausen et al., 1940; Ehrendorfer, 1973; 
Guo et al., 2004). Although the genus has a predominately Old World distribution, phylogenetic studies indicate that an autotetraploid lineage of the circumpolar A. millefolium aggregate colonized North America approximately 1 Mya (Guo et al., 2005; Ramsey, unpublished data). In a rapid radiation, these tetraploids evolved a diversity of ecological races (widespread across North America) as well as local occurrences of autohexaploids (restricted to the Pacific states and provinces of the United States and Canada) (Clausen et al., 1940, 1948; Lawrence, 1947; Tyrl, 1975). Tetraploids $(2 n=4 x=36)$ and hexaploids $(2 n=6 x=54)$ are regionally sympatric along the Pacific coast and do not have distinguishing morphological characteristics. The cytotypes moreover lack fixed differences across $3.5 \mathrm{~kb}$ of non-coding cpDNA, and appear to have diverged very recently ( $<100000$ years ago) (Ramsey, unpublished data). Here, I reference tetraploid and hexaploid cytotypes as A. borealis, based on the epithet ascribed in 1832 by Bongard.

The formation of autohexaploids in tetraploid populations occurs via unilateral sexual polyploidization, through the union of reduced $(n=2 x)$ and unreduced $(2 n=4 x)$ gametes (Bingham, 1968; Hornsey, 1973; see Ramsey and Schemske, 1998). Tyrl (1975) found that tetraploid $A$. borealis produced occasional unreduced pollen grains - identifiable by their large size - and hypothesized that hexaploid $A$. borealis formed via unreduced pollen production in tetraploid populations. This study was conducted in two tetraploid populations occurring in grasslands on the Pacific coast of California. As in other grasslands in this region, extensive cytotype surveys based on chromosome counts of reproductive adults and flow cytometry of seedlings identified only tetraploid individuals, despite the occurrence of hexaploids in nearby dune habitats (Ramsey, unpublished data). The two study populations were representative of tetraploids in the region, and were selected at random from a larger set of tetraploid study sites under ecological investigation. The study populations were separated by $50 \mathrm{~km}$, and are hereafter identified as 'site 1' and 'site 2 '.

\section{Cytology}

Cytological analyses of microsporogenesis and mature pollen were made on reproductive plants at site 1 during the summers of 1997 and 1998. Unopened florets were fixed in modified Carnoy's solution (60\% chloroform, $30 \%$ ethanol, $10 \%$ acetic acid), stored in $70 \%$ ethanol and stained in Snow's carmine, following Tyrl (1975). Stained anthers were mounted in Hoyer's medium and analyzed at $\times 400$ magnification on a compound microscope. Developing pollen was cytotyped at the first post-meiotic mitosis. Mature pollen from newly opened florets was mounted on a glass slide in a 1:2 mixture of aniline blue and Hoyer's medium. Pollen was viewed at $\times 100-400$ on a compound microscope, and measured with an ocular micrometer.

\section{Measuring unreduced pollen production}

In 1997 and 1998, the rate of unreduced pollen production was estimated at sites 1 and 2. Sampling was performed during periods, 20 June-2 August 1997 and 20 June-10 August 1998, that correspond to the peak flowering phenology of these populations. Seventy-five randomly selected plants were sampled each year at site 1 , whereas 50 plants were surveyed at site 2. Excavation of established plants in the study area revealed that genets spread over a distance of $<2 \mathrm{~m}$, and sampled individuals were separated by a minimum of $5 \mathrm{~m}$ to ensure sampling of different genets. Pollen from five randomly selected capitula per individual was mounted on a glass slide in a 1:2 mixture of aniline blue and Hoyer's medium. Samples were evaluated at $\times 400$ on a compound light microscope. The frequency of unreduced pollen in a sample was determined by tallying the total number of 'big' and 'jumbo' pollen (see below), and dividing by total pollen number. The latter was estimated from six to eight random 'transects' made across the slide. In total, more than 1.5 million pollen grains were screened, with an average of 6032 per sampled plant (s.e. $=247$, range 1494-15969). To compare the frequency of unreduced pollen between sites and years, data were rank transformed and analyzed with two-factor analysis of variance (ANOVA).

To evaluate the consistency of unreduced pollen production by individual plants, at site 1 randomly selected 'high' and 'low' unreduced pollen producers were re-sampled 10-20 days after initial screening. The frequency of unreduced pollen produced by re-sampled high and low producers was compared with MannWhitney U-tests.

\section{Neopolyploid formation}

Surveys identified several individuals from site 1 that produced substantial $(>5 \%)$ unreduced pollen (see Results), and these were used as male parents in experimental crosses with randomly selected female parents. Two high producers discovered in the field in June 1998 were used to pollinate nearby plants. Another high producer was identified in the greenhouse and used to pollinate other site 1 plants in greenhouse culture in May 1998. Tetraploid and hexaploid cytotypes of $A$. borealis are self-incompatible (Hiesey and Nobs, 1970; Ramsey, unpublished data), but pollen flow from adjacent plants could contaminate pollination treatments. Cages constructed from mesh-covered chicken wire were placed around the inflorescence of female parents to prevent insect visitation. Unpollinated control plants were also caged. Pollen was transferred using velvet fabric cut into $1 \mathrm{~cm}$ squares and glued onto toothpicks. Pollinations were performed 4-6 times per week between 1500 and 2000 hours, when most stigmas had fully emerged and were receptive to pollen transfer (Ramsey, personal observations). Crosses were continued until all florets of female parents had opened, and seeds were harvested 8-10 weeks later. As a comparison to the progeny of the unreduced pollen producers, I also collected seeds from 15 randomly selected, open-pollinated maternal parents at site 1 .

Seeds from experimental crosses and open-pollinated controls were sown into potting soil in plug trays and grown at the University of Washington Botany Greenhouse (Seattle, WA, USA) in 1998 and 1999. Trays were misted daily with clear water until germination, and then irrigated 4-5 times per week in a complete nutrient solution at $100 \mathrm{ppm}$ nitrogen. Flow cytometry was used to cytotype approximately 100-200 seedlings from each experimental 
cross, and 50-100 seedlings from the randomly selected controls. As reviewed in Arumuganathan and Earle (1991), flow cytometric analysis of nuclei provides an efficient measure of DNA content, and hence ploidy level, in many plant species. Flow cytometry is now commonly used for cytotyping large samples of individuals in studies of plant evolution (e.g., Husband and Schemske, 1998; Bretagnolle, 2001).

Flow cytometry protocol was modified from Bino et al. (1992). Newly expanding leaves were placed in $2 \mathrm{ml}$ of buffer $(3.6 \mathrm{~g}$ HEPES, $2 \mathrm{ml}$ of a $0.5 \mathrm{M}$ solution of ethylenedinitrilotetraacetic acid (EDTA), $6.0 \mathrm{~g} \mathrm{KCl}, 1.2 \mathrm{~g}$ $\mathrm{NaCl}, 102.7 \mathrm{~g}$ sucrose, $2 \mathrm{ml}$ Triton X-100, $1 \mathrm{ml} \beta$-mercaptoethanol and $0.1 \mathrm{~g}$ spermine in 1.01 distilled water) and chopped for approximately $1 \mathrm{~min}$ with a fresh razor blade. The resulting slurry was passed through a syringe filter (25 mm Millipore Swinnex filter holder, Fisher Scientific Company LLC, Pittsburgh, PA, USA, SX0002500) fitted with $48 \mu \mathrm{m}$ nylon mesh (Small Parts Inc., Miami Lakes, FL, USA, B-CMN-48) to remove debris, and then centrifuged for $30 \mathrm{~s}$ at 12000 r.p.m. The nuclei pellet was resuspended in chopping buffer containing $2 \%$ propidium iodide (PI) and $0.1 \%$ RNAse A (QIAGEN Inc., Valencia, CA, USA, 19101), and filtered a second time. All samples were run on a FACScan flow cytometer (B-D Biosciences, San Jose, CA, USA) outfitted
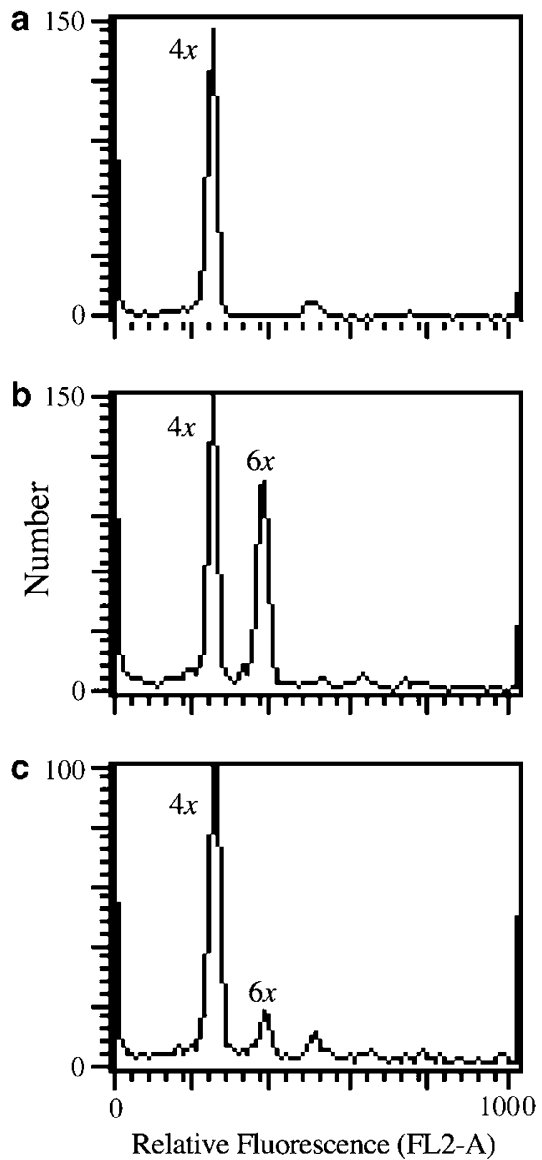

Figure 1 Flow cytometer output of relative fluorescence (FL2-A) for representative samples containing (a) only tetraploid leaf tissue, (b) approximately equal amounts of tetraploid and hexaploid leaf tissues and (c) a 5:1 ratio of tetraploid and hexaploid leaf tissues. with a blue argon laser (488 nm wavelength) in the Cell Analysis Facility, Department of Immunology, University of Washington, Seattle, USA. Samples were analysed for relative fluorescence (FL2-A), which was summarized as a frequency histogram using the CellQuest ${ }^{\mathrm{TM}}$ software package (Becton-Dickinson and Company, Franklin Lakes, NJ, USA, v. 3.3).

Tetraploids comprised the majority of the progeny of experimental crosses and the randomly selected control parents, so a pooling protocol (Bretagnolle, 2001) was used to facilitate analysis. Hexaploid nuclei (6C) have $50 \%$ greater DNA content than tetraploid nuclei (4C), and in pooled samples were unambiguously distinguished from 8C peaks generated by endopolyploidy, mitosis and doublets (Figure 1). In experimental sample preparations, hexaploid peaks were distinguished from tetraploid peaks when hexaploid leaf tissue comprised only $10-20 \%$ of a sample (Figure 1). As a conservative method, I combined leaves from three seedlings to form samples. In those cases in which samples contained both $4 x$ and $6 x$ peaks, contributing unknowns were cytotyped individually. External controls consisted of samples pooling leaf tissue of known tetraploid and hexaploid individuals and were run after every 5-10 samples.

For the progeny of experimental crosses, a $\chi^{2}$-test was used to compare the observed occurrence of neopolyploids with the value expected from the frequency of unreduced pollen produced by the siring parent.

\section{Results}

Cytological characteristics of unreduced pollen

Study of the first post-meiotic mitoses in pollen identified reduced $(n=2 x=18)$ as well as unreduced 'big' $(2 n=4 x=36)$ and 'jumbo' $(4 n=8 x=72)$ grains. The $2 n$ pollen were larger than reduced grains, and $4 n$ pollen were larger than $2 n$ grains (Figure 2). Analysis of several plants with substantial production of unreduced pollen indicated that diameters of cytotyped $n=18$ and $2 n=36$ grains differed significantly (e.g., paternal parent 3: mean 19.0 vs $27.5 \mu \mathrm{m}, N=16$, Mann-Whitney $U$-test, $Z=-3.440, P=0.0006)$ and exhibited nonoverlapping distributions. Reduced and unreduced pollen were also distinguished by overall shape. Unreduced grains were typically four-pored and round, whereas reduced grains were three-pored and more triangular (Figure 2).

Measurement of pollen grains at different stages of development - from undehisced anthers and open florets - revealed bi- and tri-modal size distributions. Figure 3 shows the distribution of pollen diameter from representative samples. The size ratio of normal vs ' $\mathrm{big}^{\prime}$ pollen was similar to the ratio of cytotyped reduced and $2 n$ pollen (e.g., diameter ratios of $1: 1.45$ vs 1:1.42). The diameter of 'jumbo' pollen was $\sim 2$ times than those of normal pollen, as observed in comparisons of cytotyped grains (Figures 2 and 3). Hereafter, size is used to infer ploidy level of pollen grains, with normal pollen corresponding to reduced grains $(n=2 x)$ and 'big' and 'jumbo' pollen corresponding to $2 n$ and $4 n$ grains, respectively.

\section{Frequency of unreduced pollen}

Production of unreduced pollen did not differ statistically between study sites or years (two-factor ANOVA, 


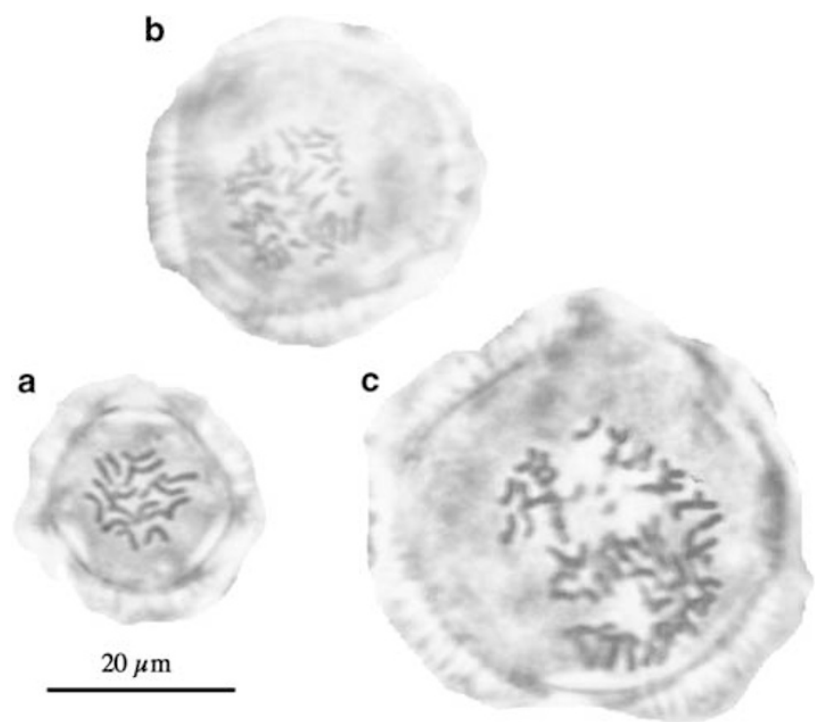

Figure 2 Photomicrographs of the first post-meiotic mitoses in (a) reduced $(n=2 x)$, (b) unreduced 'big' $(2 n=4 x)$ and (c) 'jumbo' $(4 n=8 x)$ pollen grains.
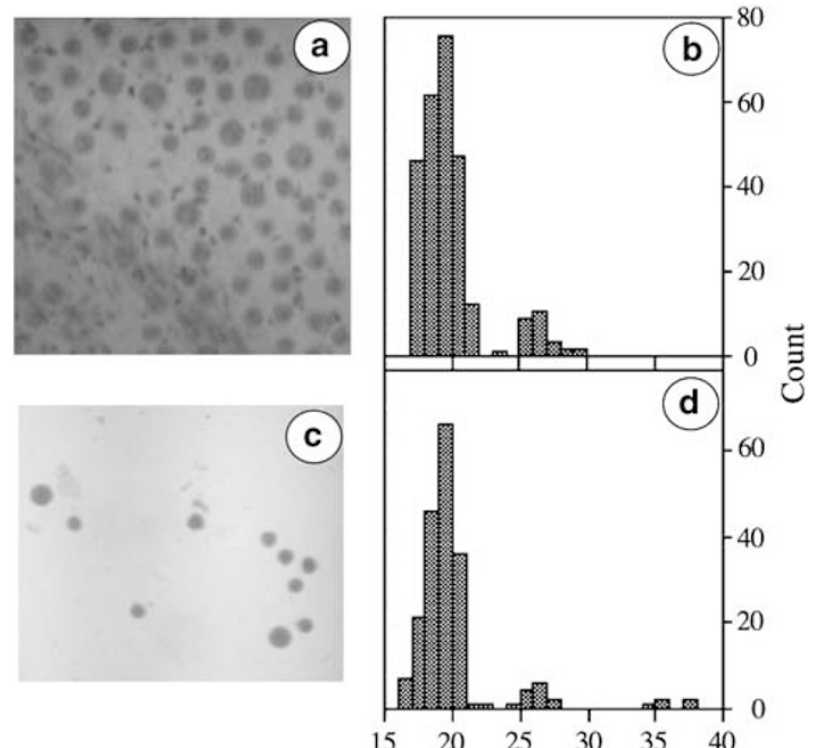

Pollen diameter $(\mu \mathrm{m})$
Figure 3 Sizes of pollen sampled at different stages of development. (a) Photomicrograph of undehisced anther (stained in carmine) (b) Diameters of pollen inside a single anther. (c) Photomicrograph of pollen from freshly dehisced anthers (stained in aniline blue). (d) Diameters of pollen collected from five open florets of one plant.

Table 1 Mean, range and variability of unreduced pollen production per plant in two study populations

\begin{tabular}{rrlllll}
\hline \multicolumn{5}{c}{ Site Year Mean (\%)Range (\%) } & s.e. Frequency & 2n pollen producers $(\%) \mathrm{N}$ \\
\hline 1 & 1997 & 0.161 & $0-6.4$ & 0.089 & 34.7 & 75 \\
& 1998 & 0.538 & $0-15.8$ & 0.286 & 49.3 & 75 \\
& & & & & & 50 \\
2 & 1997 & 0.030 & $0-0.4$ & 0.010 & 50.0 & 50 \\
\hline & 1998 & 0.130 & $0-2.5$ & 0.061 & 42.0 & \\
\hline
\end{tabular}

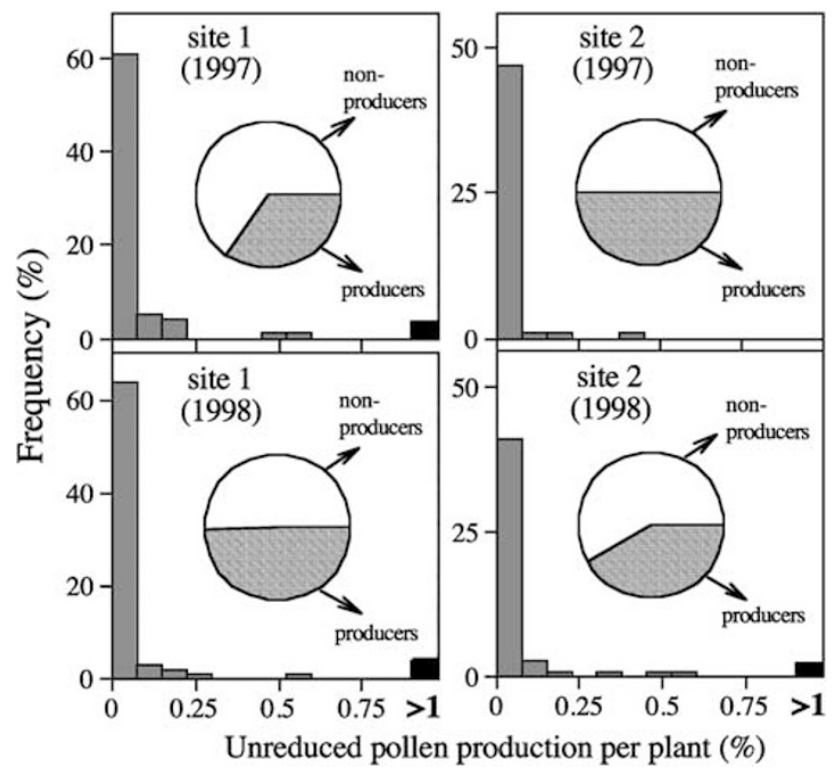

Figure 4 Distribution of unreduced pollen production per plant (histograms) and proportion of individuals producing at least occasional unreduced pollen (inset pie diagrams) for randomly selected plants at sites 1 and 2 in 1997 and 1998.

$P>0.40$ ). The mean frequencies were $0.161 \%$ (1997) and $0.538 \%$ (1998) at site 1 vs $0.030 \%$ (1997) and $0.130 \%$ (1998) at site 2, with an overall average of $0.242 \%(N=250$ plants) (Table 1). These surveys revealed a pattern in which most individuals produced few or no unreduced pollen, whereas a few plants generated substantial frequencies (Figure 4). Production of at least occasional unreduced pollen was observed in one-third to one-half of sampled plants (Figure 4). Eight individuals were found to produce $\geqslant 1 \%$ unreduced pollen, with observed frequencies of $1.9,2.1,6.4,7.0,13.2$ and $15.8 \%$ (site 1 ) as well as 1.7 and $2.5 \%$ (site 2).

The majority of unreduced grains were 'big' $2 n$ pollen rather than 'jumbo' $4 n$ pollen. The overall frequency of the latter was $0.006 \%$, a fraction of the mean occurrence of $2 n$ pollen $(0.236 \%)$. Most $4 n$ pollen was produced by a few individuals that also tended to generate a high frequency of $2 n$ pollen (data not shown). For example, nearly one-quarter of all $4 n$ pollen observed were produced by an individual at site 2 with $0.243 \% 2 n$ pollen and $0.344 \% 4 n$ pollen.

Resampling of high and low producers at site 1 indicated consistency of unreduced pollen formation throughout the flowering season (Figure 5). For example, second samples from high and low producers differed significantly in 1997 (mean 0.946 vs $0.038 \%, N=16$, Mann-Whitney $U$-test, $Z=-2.633, P=0.0085)$ and 1998 (mean 3.896 vs $0.019 \%, N=16$, Mann-Whitney $U$-test, $\mathrm{Z}=-3.411, P=0.0006)$.

\section{Unreduced pollen and neopolyploid formation}

The occurrence of neopolyploids was examined in progeny sired by three site 1 unreduced pollen producers. Paternal parents 1 and 2 averaged 8.6 and $15.8 \% 2 n$ pollen, respectively, and no $4 n$ pollen production was observed. Paternal parent 3 averaged $27.3 \% 2 n$ pollen 
and $7.2 \% \quad 4 n$ pollen. The progeny sired by these tetraploids was thus hypothesized to include neohexaploids $(n=2 x+2 n=4 x \Rightarrow 6 x)$ and neodecaploids $(n=2 x+$ $4 n=8 x \Rightarrow 10 x)$ in addition to tetraploids $(n=2 x+$ $n=2 x \Rightarrow 4 x)$.

Experimental crosses involving the high unreduced pollen producers yielded abundant seed ( $>200$ seeds per cross), whereas caged unpollinated controls generated no seeds. Seeds produced by crosses appeared normal, giving no indication of containing shriveled embryos or endosperm. Germination rates of seeds from experimental crosses and open-pollinated controls were similar, typically $>90 \%$ (Ramsey, personal observations).

A total of 774 progeny of the experimental crosses were cytotyped, revealing three neohexaploids from two of the six crosses (Table 2). No neodecaploids were discovered. For each cross, the observed frequency of neopolyploids was significantly less than that expected from the proportion of unreduced pollen produced by the siring parent (Table 2).

\section{Overall rate of neopolyploid formation}

Flow cytometry identified four neohexaploids among 856 tetraploid seedlings from 15 randomly selected
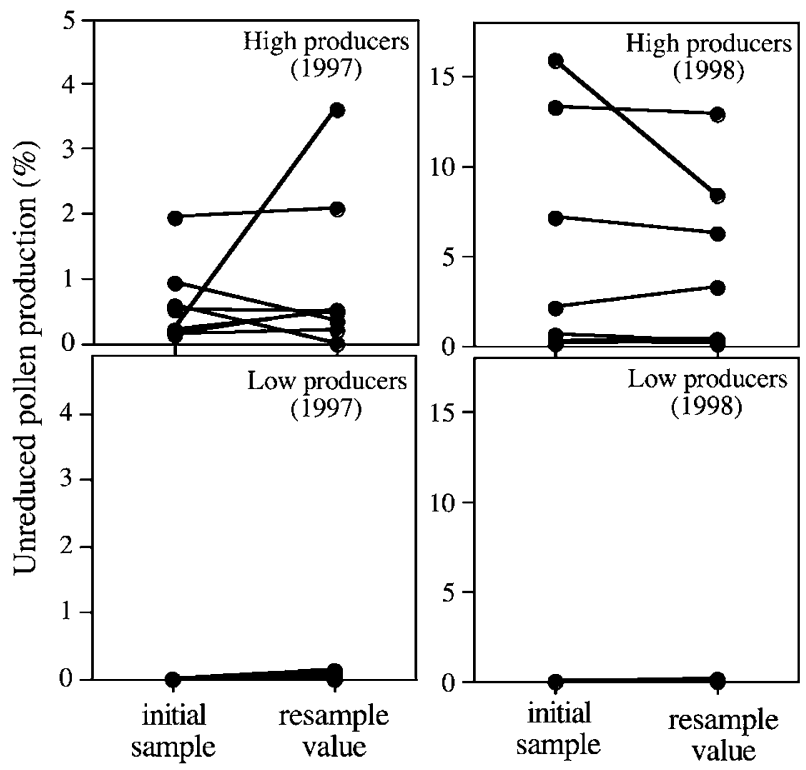

Figure 5 Production of unreduced pollen by resampled high producers $(N=8)$ and low producers $(N=8)$ at site 1 in 1997 and 1998. maternal parents at site 1 . The neohexaploids were generated by different maternal parents. No neodecaploids were found. The overall rate of neohexaploid formation for the progeny of experimental crosses $(N=774)$ and random maternal controls $(N=860)$ was $0.428 \%$, or one in $233(N=1634)$.

\section{Discussion}

\section{Size of reduced and unreduced pollen}

In $A$. borealis, unreduced pollen are easily distinguished from reduced pollen on the basis of size (Figures 2 and 3). This phenomenon is known from other taxa and reflects the increased size of nuclei containing extra sets of chromosomes (Bretagnolle and Thompson, 1995). The size ratio of reduced and unreduced pollen in $A$. borealis $(\sim 1: 1.42)$ is similar to that reported in other species (Tyagi, 1988; Orjeda et al., 1990), suggesting a general guideline for screening unreduced pollen. However, reduced and unreduced pollen sometimes lack clearcut size differences in grasses and other wind-pollinated species (Li et al., 1964; Maciera et al., 1992).

In contrast to the utility of size in distinguishing reduced and unreduced gametes, pollen size is generally a weak indicator of plant ploidy level in natural populations (Gould, 1957; Solbrig, 1965; but see Bennett, 1972). It is important to note that unreduced gametes originate as meiotic aberrations, failed cytokineses and post-meiotic fusions, whereas the reduced pollen of polyploid individuals is produced by a normal developmental process in a plant that happens to have more chromosomes. Moreover, the size and shape of reduced pollen generated by polyploids may be subject to strong selection in natural populations, whereas unreduced gametes - the product of rare mutational events - are presumably not. For these reasons, it is perhaps unsurprising to find a different relationship between ploidy and size of reduced and unreduced gametes produced by diploids, and of reduced gametes produced by diploids and polyploids.

Production of unreduced pollen in natural populations Unreduced pollen production in $A$. borealis had a low mean value $(<1 \%)$, but displayed a leptokurtic distribution that included some individuals with high levels of production. This pattern is typical of agricultural species (Bretagnolle and Thompson, 1995; Ramsey and Schemske, 1998) and consistent with recent studies in natural plant systems. For example, Bretagnolle (2001)

Table 2 Observed and expected occurrences of neopolyploids among progeny sired by tetraploid unreduced pollen producers

\begin{tabular}{|c|c|c|c|c|c|c|c|c|}
\hline \multicolumn{2}{|c|}{ Parent } & \multirow[b]{2}{*}{$\mathrm{N}$} & \multicolumn{2}{|c|}{ No. neohexaploids } & \multicolumn{2}{|c|}{ No. neodecaploids } & \multicolumn{2}{|c|}{$\chi^{2}$} \\
\hline Paternal & Maternal & & Obs. & Exp. & Obs. & Exp. & $\chi^{2}$ & $\mathrm{P}$ \\
\hline \multirow[t]{2}{*}{1} & A & 142 & 2 & 12.2 & - & - & 9.329 & 0.002 \\
\hline & $\mathrm{B}$ & 210 & 0 & 18.1 & - & - & 19.807 & $<0.0001$ \\
\hline \multirow[t]{2}{*}{2} & $\mathrm{C}$ & 109 & 1 & 17.2 & - & - & 18.117 & $<0.0001$ \\
\hline & $\mathrm{D}$ & 102 & 0 & 16.1 & - & - & 19.118 & $<0.0001$ \\
\hline \multirow[t]{2}{*}{3} & E & 89 & 0 & 24.3 & 0 & 6.4 & 46.866 & $<0.0001$ \\
\hline & $\mathrm{F}$ & 122 & 0 & 33.3 & 0 & 8.8 & 64.283 & $<0.0001$ \\
\hline
\end{tabular}


examined production of big pollen by 174 individuals in two natural populations of diploid Anthoxanthum alpinum. Mean production was $\sim 1 \%$, but 11 individuals were found to produce $>5 \%$ big pollen (Bretagnolle, 2001). Maciera et al. (1992) and de Haan et al. (1992) used experimental interploidy crosses to evaluate the production of unreduced pollen and eggs in diploid Dactylis glomerata. Survey of $\sim 100$ individuals from nine subspecies indicated an average frequency of $0.98 \% 2 n$ pollen and $0.49 \% 2 n$ eggs, including four individuals with $>5 \%$ unreduced gametes. As a general rule, it appears that approximately $0.1-2.0 \%$ of the gametes in a nonhybrid population are expected to be unreduced. It is important to note, however, that interspecific F1 hybrids often produce substantially more unreduced gametes than nonhybrid plants (Grant, 1952; Ramsey and Schemske, 1998).

In this study, $2 n$ pollen was 40 -fold more frequent than $4 n$ pollen. Production of $4 n$ pollen is documented in alfalfa, corn and peas, and generally involves a failure of cytokinesis following meiosis (Pfeiffer and Bingham, 1983). Additional cytological study is needed to understand the mechanisms underlying $2 n$ and $4 n$ pollen production in Achillea.

Analyses of agricultural species indicate that genic factors typically underlie gametic nonreduction (Bretagnolle and Thompson, 1995). Several observations point to a possible genetic basis of unreduced pollen production in $A$. borealis. First, production of unreduced pollen by individuals was stable through the season (Figure 5). Second, sampling of multiple stems on individual plants revealed similar frequencies of unreduced pollen across ramets of a genet (Ramsey, personal observations). For example, field production by four ramets of paternal parent 1 (Table 2) ranged from 7.7 to $10.0 \%$, much higher than background rates of unreduced pollen production at this site. Third, the progeny of unreduced pollen producers exhibited high levels of unreduced pollen production (Ramsey, personal observations).

No significant variation in the frequency of $2 n$ pollen was detected between study sites or years, suggesting that seasonal and edaphic environmental factors do not grossly affect gametic nonreduction in this system. However, statistical power to detect differences in the occurrence of rare mutational events is low, and environmental factors may play a general role across sites and years in promoting unreduced pollen production. For example, research in agricultural systems points to the importance of temperature in affecting meiotic processes (McHale, 1983), and preliminary experiments highlight potential temperature effects in $A$. borealis (Ramsey, unpublished data). Twenty plants collected as cuttings from site 1 in spring 1997 were grown in a growth chamber that cycled temperatures from $6^{\circ}$ to $30^{\circ} \mathrm{C}$ on a $6 \mathrm{~h}$ cycle. Unreduced pollen production was substantially greater in the temperature treatment than in the field. Specifically, 20 of 20 plants in the temperature treatment generated unreduced pollen (vs 26 of 75 in the field), with an average frequency of $0.422 \%$ (vs $0.161 \%$ in the field).

Role of unreduced pollen in neopolyploid formation

The observed occurrences of neohexaploids in experimental crosses were much less than the frequencies expected on the basis of the $2 n$ pollen produced by the siring parents (Table 2). Although paternal parent 3 was found to produce considerable $4 n$ pollen (Table 2), no neodecaploids were found in its progeny. Moreover, the frequency of neohexaploids in experiment crosses $(0.388 \%)$ was similar to background frequency of neohexaploids observed in randomly selected, openpollinated maternal sibships $(0.465 \%)$. It is not possible to unambiguously identify the cytological origin of the neopolyploids observed in this study, but the data suggest that unreduced pollen play a limited role in unilateral sexual polyploidization. The most likely mechanism responsible for neohexaploid formation is unreduced eggs.

Although there are no published analyses of the relative contributions of unreduced pollen and eggs to polyploid formation, evaluation of diploid-tetraploid crosses reveals that unreduced eggs are more likely to contribute to unilateral sexual polyploidization. For example, in a survey of the frequency of triploid formation from diploid-tetraploid crosses, Ramsey and Schemske (1998) found triploid formation in 10 of 17 studies of $4 x \times 2 x$ crosses (female $\times$ male) vs six of 19 studies of $2 x \times 4 x$ crosses. Ten of 11 studies reporting triploid formation found higher rates in $4 x \times 2 x$ crosses than $2 x \times 4 x$ crosses (Ramsey and Schemske, 1998). Hence, diploid eggs are generally more likely than diploid pollen to generate viable seeds containing triploid embryos. Formation of neotriploids in diploid populations, and neohexaploids in tetraploid populations, proceeds in an analogous fashion to these interploidy crosses.

Two factors could be responsible for the inefficiency of unreduced pollen. First, unreduced grains may compete poorly with reduced pollen. Although unreduced gametes are expected to express less genetic load and hence be more vigorous than reduced gametes, there is evidence that diploid and polyploid pollen are less viable than haploid counterparts. For example, the diploid pollen of autotetraploid maize and lettuce exhibit reduced growth rates leading to reduced fertility of inter- and intracytotype crosses (Randolph, 1935; Einset, 1944). Diploid and polyploid gametes may be negatively affected by increased cell size, gene dosage and other effects of increased ploidy. Moreover, diploid and polyploid gametes generated by gametic nonreduction and normal meiosis in polyploids are often aneuploid, which may reduce performance relative to euploid haploid grains (Ramsey and Schemske, 1998, 2002).

A second cause of the inefficiency of unreduced pollen relates to fertilization and seed development. In most diploid taxa, the triploid endosperm is generated by fusion of two ovule nuclei with one pollen generative nucleus. Seeds containing neotriploid embryos produced by unreduced pollen thus have tetraploid endosperm, whereas seeds generated by unreduced eggs usually contain pentaploid endosperm. Ploidy ratios between embryo, endosperm and maternal tissue affect development and viability of seeds generated by intercytotype crosses (reviewed in Ramsey and Schemske, 1998), suggesting that endosperm ploidy may also determine the efficiency of unreduced pollen and eggs in unilateral sexual polyploidization. In the case of $A$. borealis, the embryo:endosperm ploidy ratios generated by unreduced pollen and eggs are 6:8 and 6:10, respectively, 
whereas normal tetraploid-tetraploid crosses have a ratio of $4: 6$

In this study, seeds from experimental crosses were well-filled and exhibited high germination rates, suggesting that unreduced pollen grains were not generating inviable seeds containing neohexaploid embryos. Unreduced pollen grains stain darkly in aniline blue, and germinability of unreduced and reduced pollen grains were similar in in vitro assays (Ramsey, personal observations). Thus, the barrier to unilateral sexual polyploidization in this system is most likely to involve competitive interactions in the pistils of tetraploid plants.

\section{Rates of neopolyploid formation}

The overall rate of neohexaploid formation by tetraploid A. borealis at site 1 was $0.428 \%$, or 1 in $233(N=1634)$. This frequency is similar to rates of triploid formation via unilateral polyploidization in diploid populations (Ramsey and Schemske, 1998). For example, triploids comprised $0.279 \%$ of the progeny of diploid apple cultivars (Einset, 1952), and $0.212 \%$ of seedlings in natural populations of diploid A. alpinum (Bretagnolle, 2001). Unlike triploids, however, hexaploids have an evennumbered ploidy level and are meiotically stable. Neohexaploids are thus akin to neotetraploids, but form at a faster rate because their production involves unilateral rather than bilateral sexual polyploidization. On the basis of analyses of triploid block, triploid meiosis and rates of gametic nonreduction, Ramsey and Schemske (1998) estimated that the rate of autotetraploid formation per generation in diploid populations is similar to the average per locus genic mutation rate $\left(10^{-5}\right)$. The observed rate of neohexaploid formation in A. borealis $(0.00428)$ is similar to the square root of the estimated rate of tetraploid formation in outcrossing populations (0.00578), as would be expected.

The production of neohexaploids from tetraploids has been noted in several agricultural systems. For example, the progeny of autotetraploid Beta vulgaris contained 2\% hexaploid cytotypes (Hornsey, 1973), whereas $1 \%$ of the progeny of autotetraploid alfalfa were found to be hexaploid (Bingham, 1968). There is circumstantial evidence of local hexaploid formation in natural tetraploid plant populations (Dewey and Asay, 1975). For example, Clausen et al. (1940) discovered a single hexaploid genotype in an otherwise-tetraploid population of Artemisia ludoviciana, and speculated that it seems plausible to suppose that it arose as an autopolyploid from the tetraploid ludoviciana through the union of a diploid and a haploid gamete. Occasional production of diploid gametes is to be expected in climates so extreme as the alpine' (p. 336). Neohexaploid formation is probably a common event in tetraploid populations (Ramsey and Schemske, 1998).

\section{General conclusions}

Unreduced gametes are widely regarded as an important mechanism of polyploid formation, but there are few investigations relating the production of neopolyploids to specific cytological mechanisms (Bretagnolle and Thompson, 1995; Ramsey and Schemske, 1998). In $A$. borealis and other species, unreduced pollen has been hypothesized to drive polyploid evolution via unilateral sexual polyploidization. This study finds unreduced pollen to be a common meiotic aberration with intriguing characteristics, including possible genetic and environmental components. However, experimental crosses suggest that unreduced pollen play a limited role in neopolyploid formation. Additional research is needed to evaluate the mechanisms responsible for generating the neohexaploids occurring at high frequencies in the progeny of tetraploids, but unreduced eggs are the most likely source. Like other composites, $A$. borealis is poorly suited for studies of megasporogenesis because florets contain a single ovule. Indirect studies of unreduced eggs could be pursued by measuring rates of neohexaploid formation across maternal parent genotypes crossed to tester male parents.

In spite of the inefficiency of unreduced pollen, rates of neohexaploid formation in $A$. borealis are two orders of magnitude greater than typical estimates of genic mutation rates. The high rates observed in this and other systems are striking given the profound genetic and phenotypic changes that accompany polyploid formation (Ramsey and Schemske, 2002), and the ecogeographic differences distinguishing established cytotypes in natural complexes (Müntzing, 1936; Lewis, 1980). Comparisons of tetraploid and neohexaploid genotypes identified in this project indicate that in a single generation, polyploidization produced a substantial transition in phenotypic characteristics, including several apparent adaptations to the dune environments currently inhabitated by established hexaploids (Ramsey, unpublished data). These results underscore the role of chromosome evolution as a mechanism of phenotypic differentiation.

\section{Acknowledgements}

I thank Doug Schemske for his advice and encouragement through all stages of this research. Toby Bradshaw, Brian Husband, Dick Olmstead and two anonymous reviewers provided helpful suggestions on a draft of this manuscript. Chris Oakley, Dena Grossenbaucher, Kim Young and Abby Sine assisted with sample preparation. Special thanks to Ken Anderson, Jay Harris and Michelle Forys of the California Parks North Coast Redwood District for permission to conduct this research. This research was supported by grants from the National Science Foundation (dissertation improvement grant DEB-9902294), the California Native Plant Society, Sigma $\mathrm{Xi}$ and the Botanical Society of America. This material is based on work supported under a National Science Foundation predoctoral fellowship.

\section{References}

Arumuganathan K, Earle ED (1991). Estimation of nuclear DNA content of plants by flow cytometry. Plant Mol Biol Reporter 9 : 229-233.

Bennett MD (1972). Nuclear DNA content and minimum generation time in herbaceous plants. Proc $R$ Soc Lond $B$ 181: 109-135.

Bingham ET (1968). Aneuploids in seedling populations of tetraploid alfalfa, Medicago sativa L. Crop Sci 8: 571-574.

Bino RJ, de Vries JN, Kraak HL, van Pijlen JG (1992). Flow cytometric determination of nuclear replication stages in tomato seeds during priming and germination. Ann Bot 69 : 231-236. 
Bretagnolle F (2001). Pollen production and spontaneous polyploidization in diploid populations of Anthoxanthum alpinum. Biol J Linn Soc 72: 241-247.

Bretagnolle F, Thompson JD (1995). Tansley review no. 78. Gametes with the somatic chromosome number: mechanisms of their formation and role in the evolution of autopolyploid plants. New Phytol 129: 1-22.

Clausen J, Keck D, Hiesey WM (1940). Experimental studies on the nature of species. I. Effect of varied environments on western North American plants. Carnegie Inst Washington Publ 520: 1-452.

Clausen J, Keck D, Hiesey WM (1945). Experimental studies on the nature of species. II. Plant evolution through amphiploidy and autoploidy, with examples from the madinae. Carnegie Inst Washington Publ 564: 1-163.

Clausen J, Keck D, Hiesey WM (1948). Experimental studies on the nature of species. III. Environmental responses of climatic races of Achillea. Carnegie Inst Washington Publ 581: 1-129.

Coyne JA, Orr HA (2004). Speciation. Sinauer Associates: Sunderland.

De Haan A, Maciera NO, Lumaret R, Delay J (1992). Production of $2 n$ gametes in diploid subspecies of Dactylis glomerata L. 2. Occurrence and frequency of $2 n$ eggs. Ann Bot 69: 345-350.

Dewey DR, Asay KH (1975). The crested wheat grasses of Iran. Crop Sci 15: 844-849.

Ehrendorfer F (1973). New chromosome numbers and remarks on the Achillea millefolium polyploid complex in North America. Osterr Bot Z 122: 133-143.

Einset J (1944). Cytological basis for sterility in induced autotetraploid lettuce (Lactuca sativa L.). Am J Bot 31: 336-342.

Einset J (1952). Spontaneous polyploidy in cultivated apples. J Am Soc Hort Sci 59: 291-302.

Gould FW (1957). Pollen size as related to polyploidy and speciation in the Andropogon saccharoides-A. barbinodis complex. Britonnia 9: 71-75.

Grant V (1952). Cytogenetics of the hybrid Gilia millefoliata $\times$ achilleaefolia. I. Variations in meiosis and polyploidy rate as affected by nutritional and genetic conditions. Chromosoma $\mathbf{5}$ : 372-390.

Grant V (1981). Plant Speciation, 2nd edn. Columbia University Press: New York.

Guo Y-P, Ehrendorfer F, Samuel R (2004). Phylogeny and systematics of Achillea (Asteraceae-Anthemidae) inferred from nrITS and plastid trnL-F DNA sequences. Taxon 53: 657-672.

Guo Y-P, Saukel J, Mittermayr R, Ehrendorfer F (2005). AFLP analyses demonstrate genetic divergence, hybridization, and multiple polyploidization in the evolution of Achillea (Asteraceae-Anthemidae). New Phytol 166: 273-290.

Harlan JR, deWet JMJ (1975). On O Winge and a prayer: the origins of polyploidy. Bot Rev 41: 361-390.

Hiesey WM, Nobs M (1970). Genetic and transplant studies on contrasting species and ecological races of the Achillea millefolium complex. Bot Gaz 131: 245-259.

Hornsey KG (1973). The occurrence of hexaploid plants among autotetraploid populations of sugar beet (Beta vulgaris L.), and the production of tetraploid progeny using a diploid pollinator. Caryologia 26: 225-228.

Husband B, Schemske DW (1998). Cytotype distribution in a diploid-tetraploid contact zone in Chamerion (Epilobium) angustifolium. Am J Bot 85: 1688-1694.

Lawrence WE (1947). Chromosome numbers in Achillea in relation to geographic distribution. Am J Bot 34: 538-545.

Levin DA (1983). Polyploidy and novelty in flowering plants. Am Nat 122: 1-25.

Lewis WH (1980). Polyploidy in species populations. In: Lewis WH (ed). Polyploidy: Biological Relevance. Plenum Press: New York. pp. 103-144.

Li HW, Kang KKS, Ho H-C (1964). Cytogenetical studies of Oryza sativa L. and its related species. Bot Bull Acad Sinica 5: 142-153.

Maciera NO, De Haan A, Lumaret R, Billon M, Delay J (1992). Production of $2 n$ gametes in diploid subspecies of Dactylis glomerata L. 1. Occurrence and frequency of $2 n$ pollen. Ann Bot 69: 335-343.

Masterson J (1994). Stomatal size in fossil plants: evidence for polyploidy in majority of angiosperms. Science 264: 421-424.

McHale NA (1983). Environmental induction of high frequency $2 n$ pollen formation in diploid Solanum. Can J Genet Cytol 25: 609-615.

Müntzing A (1936). The evolutionary significance of autopolyploidy. Hereditas 21: 263-378.

Orjeda G, Freyre R, Iwanaga M (1990). Production of $2 n$ pollen in diploid Ipomoea trifida, a putative wild ancestor of sweet potato. J Hered 81: 462-467.

Otto SP, Whitton J (2000). Polyploid incidence and evolution. Annu Rev Genet 34: 401-437.

Pfeiffer TW, Bingham ET (1983). Abnormal meiosis in alfalfa, Medicago sativa: cytology of $2 \mathrm{~N}$ egg and $4 \mathrm{~N}$ pollen formation. Can J Genet Cytol 25: 107-112.

Ramsey J, Schemske DW (1998). Pathways, mechanisms and rates of polyploid formation in flowering plants. Annu Rev Ecol Syst 29: 467-501.

Ramsey J, Schemske DW (2002). Neopolyploidy in flowering plants. Annu Rev Ecol Syst 33: 589-639.

Randolph LF 1935. Cytogenetics of tetraploid maize. J Agric Res Wash DC 50: 591-605.

Solbrig OT (1965). The California species of Gutierrezia (Compositae-Astereae). Madrono 18: 75-84.

Soltis DE, Soltis PS (1999). Polyploidy: recurrent formation and genome evolution. Trends Ecol Evol 14: 348-352.

Thompson JD, Lumaret R (1992). The evolutionary dynamics of polyploidy plants: origins, establishment and persistence. Trends Ecol Evol 7: 302-307.

Tyagi BR (1988). The mechanism of $2 n$ pollen formation in diploids of Costus speciosus (Koenig) J.E. Smith and role of sexual polyploidization in the origin of intraspecific chromosomal races. Cytologia 53: 763-770.

Tyrl RJ (1975). Origin and distribution of polyploid Achillea (Compositae) in western North America. Brittonia 27: 287-296. 